\title{
LUT
}

University

\section{Metal-insulator switching of vanadium dioxide for controlling spin-wave dynamics in magnonic crystals}

Nikitin Aleksei A., Nikitin Andrey A., Ustinov Alexey B., Komlev Andrey E., Lähderanta Erkki, Kalinikos Boris A.

This is a Author's accepted manuscript (AAM) version of a publication

published by AIP Publishing

in Journal of Applied Physics

DOI: $10.1063 / 5.0027792$

Copyright of the original publication: (c) 2020 Author(s)

Please cite the publication as follows:

Nikitin, A.A., Nikitin, A.A., Ustinov. A.B., Komlev, A.E., Lähderanta E., Kalinikos B.A. (2020). Metal-insulator switching of vanadium dioxide for controlling spin-wave dynamics in magnonic crystals. Journal of Applied Physics, vol. 128, issue 18. DOI: 10.1063/5.0027792

This is a parallel published version of an original publication.

This version can differ from the original published article. 


\title{
Metal-insulator switching of vanadium dioxide for controlling spin-wave dynamics in magnonic crystals
}

\author{
Aleksei A. Nikitin ${ }^{1 a)}$, Andrey A. Nikitin ${ }^{1}$, Alexey B. Ustinov ${ }^{1}$, Andrey E. Komlev ${ }^{1}$, Erkki \\ Lähderanta ${ }^{2}$, and Boris A. Kalinikos ${ }^{1}$ \\ ${ }^{1}$ Department of Physical Electronics and Technology, St. Petersburg Electrotechnical \\ University, 197376 St. Petersburg, Russia \\ ${ }^{2}$ Department of Physics, LUT-University, 53850 Lappeenranta, Finland \\ a) Author to whom correspondence should be addressed: aleksei.a.nikitin@gmail.com
}

\begin{abstract}
The present work focuses on the effect of vanadium dioxide $\left(\mathrm{VO}_{2}\right)$ films exhibiting a metal-insulator transition (MIT) on performance characteristics of the magnetic multilayers. It has been shown that the MIT provides a novel mechanism for controlling the microwave spin-wave dynamics in the yttrium-iron garnet (YIG) films. In particular, the low and high levels of microwave attenuation of spin waves transmitted through the YIG- $\mathrm{VO}_{2}$ bilayer has been observed due to a variation of the $\mathrm{VO}_{2}$ conductivity within a narrow temperature range. This effect has been utilized to realize fully reconfigurable magnonic crystals composed of the thickness-modulated YIG and regular $\mathrm{VO}_{2}$ films. Promising functionality of the proposed waveguiding structures arises from a controllability of wave intensity, which provides altering of the frequency response from an original band structure to a full rejection of spin waves. Numerical simulations taking into account both the YIG-film saturation magnetization and the $\mathrm{VO}_{2}$-film conductivity have confirmed the experimentally observed spin-wave dynamics. An interest in ferrite$\mathrm{VO}_{2}$ bilayers arises not only from possible practical applications, but also from a variety of fundamental scientific problems devoted to physics of wave phenomena in planar thin-film magnetic multilayers.
\end{abstract}

\section{INTRODUCTION}

In the last decade, peculiarities of the microwave spin-wave (SW) processes in diverse magnetic materials have opened new avenues in the field of magnonics. An increased research activity in this field is due to development of novel microwave devices for analog signal processing and data transmission [1-5]. Artificial spatially periodic magnetic media, called magnonic crystals (MCs), constitute one of the 
most promising building blocks for these applications. In these crystals, the formation of spectral regions, band-gaps, with prohibited wave propagation is caused by Bragg scattering.

Depending on the desired performance, the MCs are usually fabricated from ferrite or ferromagnetic materials by means of a periodic variation of any waveguiding parameter obtained by different techniques, such as chemical etching, ion implantation, metal deposition, or other. One of the widely used ways for the MC fabrication is a geometrical patterning of thin ferrite films such as yttrium-iron garnet (YIG). This is because the $\mu \mathrm{m}$-thick YIG films exhibit a low damping for propagating spin waves (SWs) and a wide tunability of their properties at microwave frequencies. The features of the MCs, such as a charge-less propagation and band structure engineering, enable one to implement them in many microwave applications including power limiters [6], magnetic field sensors [7, 8], microwave oscillators [9, 10], spin-wave logic gates [11], magnon transistors [12], and filters [13], to name a few.

Further advances in this field were achieved owing to a development of the dynamic MCs [14]. Promising functionalities of these crystals arise from the nonreciprocal behavior and dynamic controllability, which provides altering of the band-gaps from a full rejection to a full transmission. Usually two types of the dynamic MCs are distinguished, namely the current- and voltage-controlled MCs. The first one originates from a spatial variation of a magnetic field induced by an electric current in the metal wires or in the periodic screens $[11,14,15]$. The mechanisms behind the second one are divided into the two types. These are a voltage-controlled perpendicular magnetic anisotropy in ferromagnetic-dielectric heterostructures $[16,17]$ and a voltage-controlled variation of the ferroelectric layer permittivity in ferrite-ferroelectric structures [18]. Another way to produce a fully tunable MC is to use a laser radiation, which creates thermal landscapes and results in periodic modulations of the saturation magnetization [19]. Various types of the dynamic MCs are developed pursuing miniaturization of magnonic devices, tunability of their operation bands as well as reduction of switching time between $\mathrm{ON}$ and $\mathrm{OFF}$ states. Moreover, an intensive development of magnonics has 
lighted out perspectives of novel hybrid structures, where the MCs are combined with superconducting [20], ferroelectric [18, 21-26], and magnetic media [27, 28].

Another type of the novel hybrid structures was reported in [29, 30], where the microwave properties of the MCs were modified through utilizing a metal screen. The general idea of these works takes its origin from an influence of the perfectly conductive planes on the spin-wave propagation, which has been well studied as far back as the 1980s of the last century [31]. Nevertheless, many challenges in this topic are still to be addressed, and the investigations of conductivity influence on the microwave properties of the magnetic multilayers are still highly relevant [32-35]. One of the key direction inherent to the above-mentioned hybrid structures is associated with implementation of the novel approaches allowing to exploit spin-wave phenomena for more efficient microwave devices.

Recently, our theoretical works predicted promising features of the metal-insulator transition (MIT) in the heterogeneous ferrite-vanadium dioxide structures [36, 37]. Such transition is characterized with an abrupt change of the $\mathrm{VO}_{2}$ conductivity (by four or five orders of magnitude) near the phase transition temperature at ultrafast timescales [38-41]. In the current work, the experimental investigations of the heterogeneous ferrite-vanadium dioxide structures are presented for the first time. The research is focused on the application of the metal-insulator transition for controlling the microwave spin-wave dynamics of the novel heterogeneous structures.

\section{EXPERIMENTAL SETUP}

In order to clarify the spin-wave dynamics in the proposed heterogeneous structures, a regular YIG$\mathrm{VO}_{2}$ waveguiding structure without any spatial periodic modulation is investigated in parallel with the grooved $\mathrm{MC}-\mathrm{VO}_{2}$ bilayer. The investigated structures were fabricated in a sandwich-type configuration.

Fig. 1 illustrates the sketches of the experimental layered structures comprising the regular (a) and spatially periodic (b) YIG films, an attached $\mathrm{VO}_{2}$ thin-film strip, and the microstrip antennas serving for the excitation and detection of SWs in GHz frequency range. 

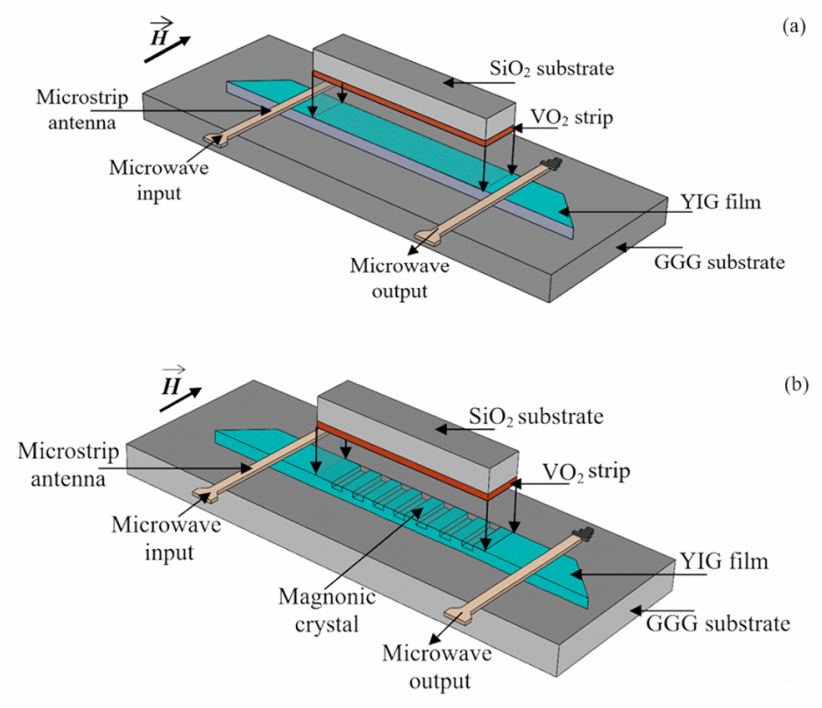

Fig. 1. Sketches of the experimental structures for (a) regular and (b) spatially periodic situations demonstrating the YIG film on the gadolinium gallium garnet (GGG) substrate and the $\mathrm{VO}_{2}$ film on the silicon dioxide $\left(\mathrm{SiO}_{2}\right)$ substrate.

The regular ferrite waveguide was fabricated in the form of the 3-cm-long and 2-mm-wide stripe of the 13.6- $\mu$ m-thick single-crystal YIG film. This film had a saturation magnetization $M_{\mathrm{s}, \mathrm{rt}}=1970 \mathrm{G}$ at the room temperature. The periodic structure was fabricated in the form of the 3-cm-long and 2-mm-wide stripe of the 5.5- $\mu \mathrm{m}$-thick single-crystal YIG film. This film had a saturation magnetization $M_{\mathrm{s}, \mathrm{rt}}=1985$ $\mathrm{G}$ at the room temperature. In order to produce a MC, the thickness of the YIG film was periodically modulated to a depth of $\delta=0.8 \mu \mathrm{m}$ using a wet chemical etching. The periodic pattern consisted of 10 parallel grooves with a width of $65 \mu \mathrm{m}$ spaced by $265 \mu \mathrm{m}$, so that the lattice constant was $330 \mu \mathrm{m}$. The both films were epitaxially-grown on the gallium gadolinium garnet (GGG) substrates of $500-\mu \mathrm{m}$ thickness. The films had the ferromagnetic resonance linewidth of $\Delta H=0.5$ Oe at $5 \mathrm{GHz}$.

A $0.55-\mu$ m-thick $\mathrm{VO}_{2}$ film was used for the both configurations. This film was deposited on a $\mathrm{SiO}_{2}$ substrate with the thickness of $500 \mu \mathrm{m}$ by a reactive DC magnetron sputtering. The sample had in-plane dimensions of $3 \times 3 \mathrm{~mm}^{2}$. The $\mathrm{VO}_{2}$ film was pressed to the one side of the regular YIG-film waveguide or to the spatial periodic surface of the MC. 
The short-circuited microstrip antennas had the width of $50 \mu \mathrm{m}$ and the length of $2.5 \mathrm{~mm}$. The antennas were positioned at the top surfaces of the YIG-film waveguides for the both configurations. The composed waveguiding structures were magnetized to saturation by an in-plane external magnetic field of 1566 Oe applied parallel to the antennas providing the conditions for excitation of the surface SWs. To accomplish the measurements, the experimental waveguiding structures were placed in a temperature-controlled probe station providing monitored heating and cooling.

Let us consider a principle of the surface $\mathrm{SW}$ propagation through the $\mathrm{YIG}-\mathrm{VO}_{2}$ structures. Initially, the wave is excited by the input microstrip antenna and propagates in the uncovered YIG film. At the border, where the YIG film is in contact with the $\mathrm{VO}_{2}$, the dispersion of the surface $\mathrm{SW}$ is changed in accordance with variation of the $\mathrm{VO}_{2}$ conductivity. The wave passes through the $\mathrm{YIG}-\mathrm{VO}_{2}$ part of the waveguiding structure and is received by the output microstrip antenna located on the uncovered YIGfilm surface. The microstrip transmission lines of $50-\Omega$ characteristic impedance fed the microstrip antennas. A vector network analyzer R\&S@ZVA40 measured the transmission characteristics of the structures.

\section{ELECTRICAL AND MAGNETIC CHARACTERIZATION}

In this Section, the temperature dependences of the $\mathrm{VO}_{2}$ conductivity and YIG film saturation magnetization are discussed. As the first step, the temperature dependences of the $\mathrm{VO}_{2}$ film conductivity were investigated. Characterization of the MIT in the $\mathrm{VO}_{2}$ film was performed by the current-voltage measurements using Keithley 2635A source meter in a temperature-controlled probe station. The measured results obtained with temperature increasing and decreasing are shown in Fig $4 \mathrm{a}$ by red squares and blue circles, respectively. 


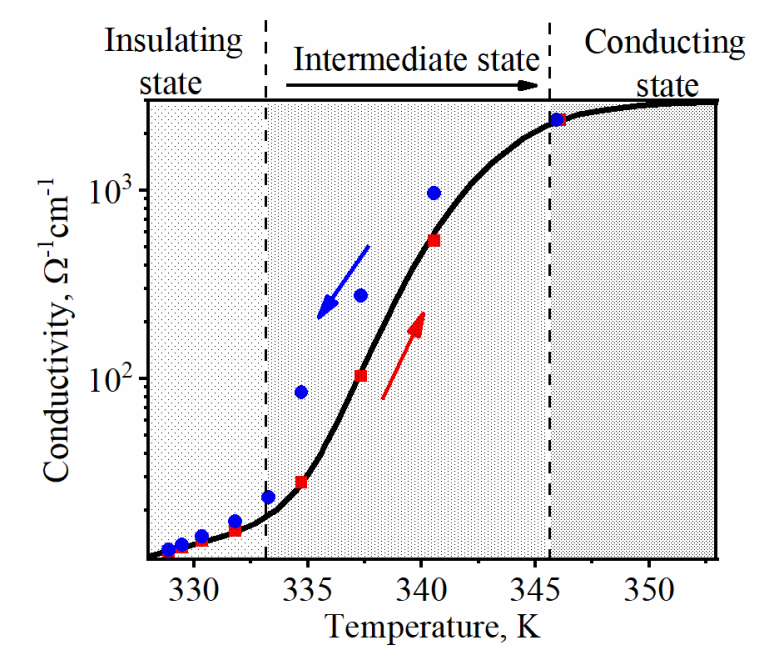

Fig.2. Temperature dependences of the $\mathrm{VO}_{2}$ conductivity. The black line is a fit described by Eq. (1).

The experimental results presented in Fig. 2 can be summarized as follows. First, the $\mathrm{VO}_{2}$ sample demonstrates a first-order metal-insulator phase transition around $T_{\mathrm{MIT}} \sim 339 \mathrm{~K}$ caused by the transformation of a monoclinic crystal structure of the sample into a tetragonal one, with a sharp (up to five orders of magnitude) increase in the conductivity. Second, the $\mathrm{VO}_{2}$ film exhibits an insulating state for $T$ below $333 \mathrm{~K}$ (area of light gray color), a conducting state (area of dark gray color) for $T$ above 346 $\mathrm{K}$, and an intermediate state between them (area of gray color). The variation of temperature within a narrow range from $333 \mathrm{~K}$ up to $346 \mathrm{~K}$ provides an abrupt change of the conductivity from $\sigma=20 \Omega^{-1}$ $\mathrm{cm}^{-1}$ up to $\sigma=1380 \Omega^{-1} \mathrm{~cm}^{-1}$. Third, the width of the hysteresis, which is attributed to different pathways of a phase transformation between the heating and cooling semi-cycles, is narrow and is estimated to be $3 \mathrm{~K}$. The range of the conductivity variation and the width of the hysteresis loop indicate small stoichiometric deviations of the produced $\mathrm{VO}_{2}$ film $[39,41]$.

Conductivity dependences obtained with temperature increasing and decreasing show similar qualitative results, but shifted by $3 \mathrm{~K}$. Owing to a small temperature shift, we restrict ourselves to investigation of the proposed structure only for the heating semi-cycle. An expression approximating the measured dependence of the $\mathrm{VO}_{2}$ conductivity with an increase in temperature has the following form: 


$$
\begin{aligned}
& \sigma(T)=S(T)+N(T), \\
& S(T)=6.605 \times 10^{-5} \times T^{3} \times \int_{-T}^{T} \frac{1}{3.079 \cdot \sqrt{2 \pi}} \times \exp \left(-\frac{(x-342.957)^{2}}{18.957}\right) d x, \\
& H(T)=-13.457 \times 10^{3}+132.409 \times T-0.434 \times T^{2}+4.751 \times 10^{-4} \times T^{3} .
\end{aligned}
$$

The temperature-dependent analytical expressions $N(T)$ and $S(T)$ were found by approximating the experimental data by the cubic parabola and modified Gaussian error function. The conductivitytemperature dependence calculated by Eq. 1 is shown in Fig. 2 by black line.

As the second step, an influence of temperature on the YIG film saturation magnetization is discussed. It is known that heating provides a reduction of a ferrite saturation magnetization. To illustrate this effect, the transmission characteristics of the free-standing regular YIG film were measured for various temperatures ranging from $298 \mathrm{~K}$ to $348 \mathrm{~K}$ (see black solid, red dashed, and blue dashed-dotted lines in Fig 3a). An increase of temperature provides a frequency downshift of the transmission characteristic. Namely, a cutoff frequency for the surface $\mathrm{SW} f_{\perp}(T)$ decreases from $f_{\perp}(298 \mathrm{~K})=6.564 \mathrm{GHz}$ to $f_{\perp}(348 \mathrm{~K})=6.396 \mathrm{GHz}$.

The cutoff frequency for the surface SW is well known and defined by the following function $f_{\perp}(T)=\sqrt{\omega_{H}\left(\omega_{H}+\omega_{M}(T)\right)} / 2 \pi$, where $\omega_{H}=\gamma \mu_{0} H_{0}, \quad \omega_{M}(T)=\gamma \mu_{0} M_{s}(T), \quad \mu_{0}=4 \pi \times 10^{-7} \mathrm{H} \cdot \mathrm{m}^{-1}$ is the vacuum permeability, $\gamma=2.8 \times 10^{10} \mathrm{~s}^{-1} \cdot \mathrm{T}^{-1}$ is the gyromagnetic ratio of electron [42]. The experimental values of the saturation magnetization were found from the function of the cutoff frequency on temperature in the following form: $M_{s}(T)=\frac{\left(2 \pi f_{\perp}(T)\right)^{2}-\omega_{H}{ }^{2}}{\gamma \mu_{0} \omega_{H}}$. The dependence $M_{s}(T)$ is shown by red dots in Fig. 3 b for 298 K, 314 K, and 348 K, respectively.

An expression approximating the experimentally measured dependence of the saturation magnetization of the YIG film on temperature ranging from $298 \mathrm{~K}$ to $348 \mathrm{~K}$ has the following form: 
$M_{s}(T)=M_{s, r t}-\eta \times\left(T-T_{r t}\right)$,

where $M_{s, r t}$ is the static magnetization at room temperature $T_{r t}=298 \mathrm{~K}$, and $\eta=313 \mathrm{~A} \mathrm{~K}^{-1} \mathrm{~m}^{-1}$. This theoretical dependence is shown by black line in Fig. 3b. This dependence is used in the further investigations, which allows to distinguish the temperature influence of the YIG magnetic properties from the direct MIT impact.
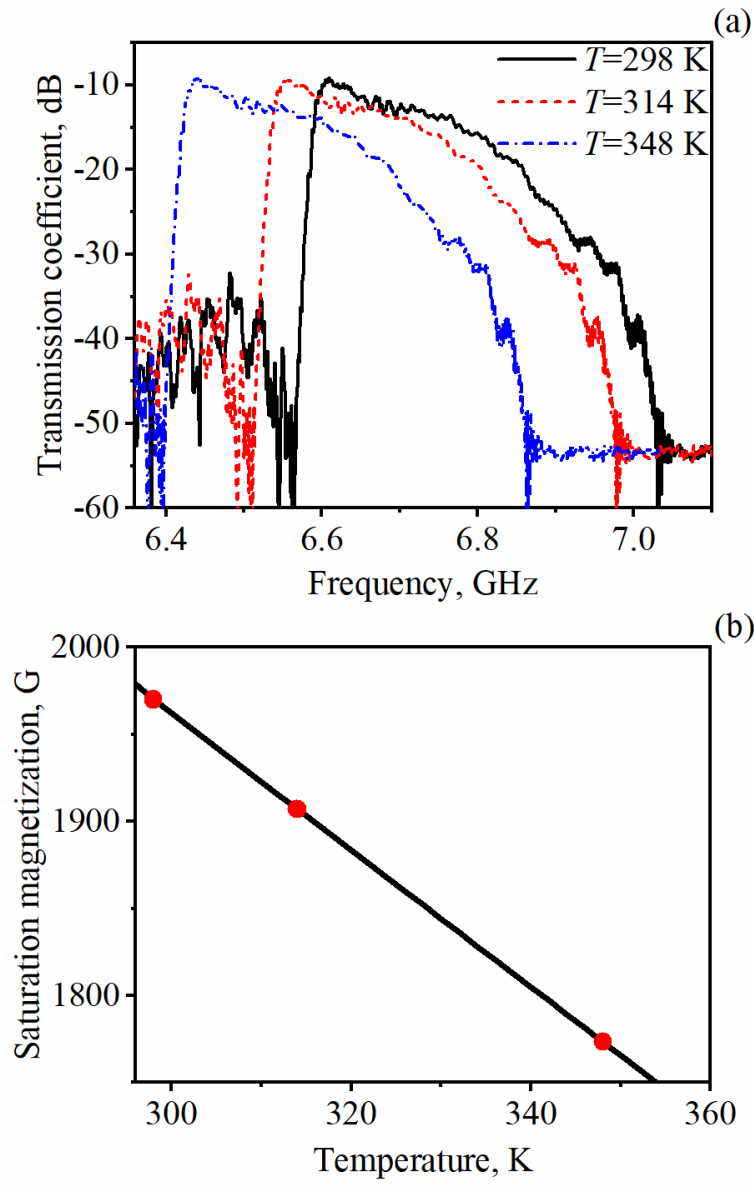

Fig. 3. (a) Transmission characteristics of the regular YIG film measured for three temperatures. (b) The saturation magnetization of the YIG film versus temperature.

IV. EXPERIMENTAL AND THEORETICAL RESULTS

(a) Microwave measurements of a regular structure composed of the $\mathrm{YIG}^{-\mathrm{VO}_{2}}$ bilayer 
In this Section, the experimental and theoretical investigations of the regular and periodic $\mathrm{YIG}-\mathrm{VO}_{2}$ waveguide structures are discussed. The transmission characteristics of the regular $\mathrm{YIG}^{-\mathrm{VO}_{2}}$ structure measured for three temperatures are presented in Fig. 4 by solid black lines. Hereinafter the frequency is normalized by $f_{\perp}$ to simplify a comparison of the obtained results.

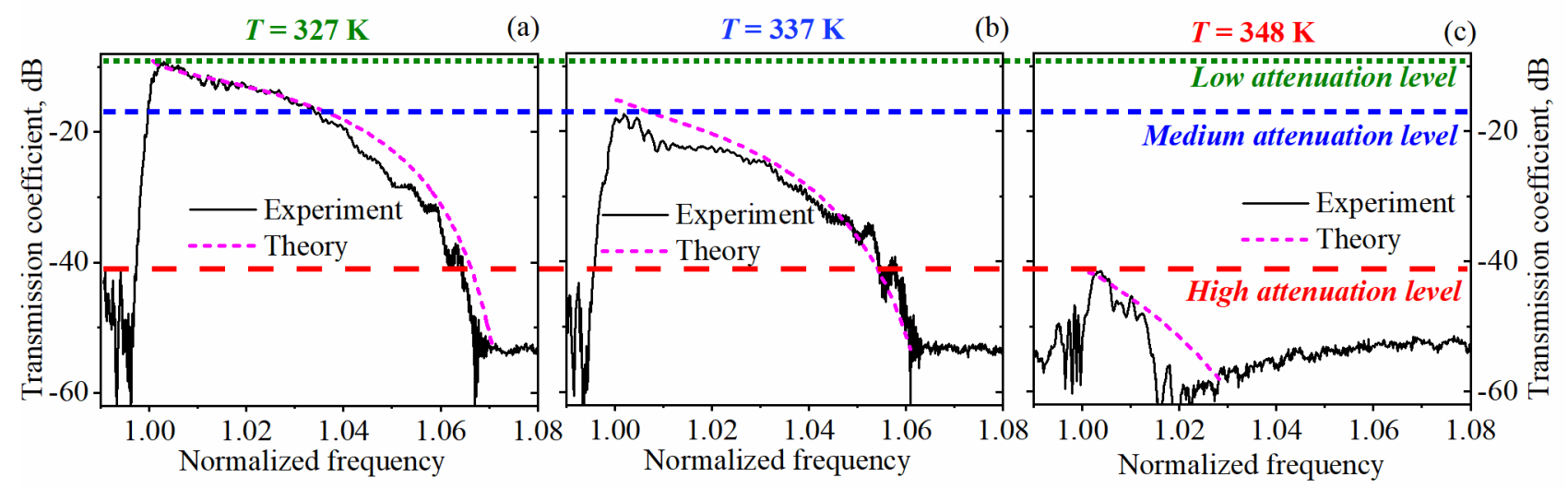

Fig. 4. Experimental (solid black lines) and theoretical (dashed magenta lines) transmission characteristics of the regular YIG-VO $\mathrm{VO}_{2}$ structure for three temperatures: (a) $T=327 \mathrm{~K}$, (b) $T=337 \mathrm{~K}$, and (c) $T=348 \mathrm{~K}$.

The experimental results shown in Fig. 4a can be explained as follows. Near room temperature the conductivity of the $\mathrm{VO}_{2}$ film is low (see Fig. 2). Therefore, the $\mathrm{VO}_{2}$ film has a negligible effect on the spin-wave propagation in the $\mathrm{YIG}-\mathrm{VO}_{2}$ bilayer in the experimental frequency range. In particular, at the initial temperature $T=327 \mathrm{~K}$, the transmission characteristics of the $\mathrm{YIG}^{\mathrm{V}} \mathrm{VO}_{2}$ bilayer and the uncovered YIG film are similar. Here, the loss slowly decreases from the minimum value of $9 \mathrm{~dB}$ (see green dotted line in Fig. 4) to about $40 \mathrm{~dB}$. A heat of the layered structure in the temperature-controlled probe station provides increasing of the $\mathrm{VO}_{2}$ conductivity (see Fig.2).

The transmission characteristic of the $\mathrm{YIG}-\mathrm{VO}_{2}$ bilayer measured at the temperature of $337 \mathrm{~K}$ is shown in Fig. $4 \mathrm{~b}$ by black solid line. As is seen, the minimum loss level equals to $18 \mathrm{~dB}$ (see blue short dash line in Fig. 4). This value is twice as high as the loss level of SWs transmitted through the bilayer 
having the insulating $\mathrm{VO}_{2}$ film at $T=327 \mathrm{~K}$. Further heating of the bilayer leads to a rapid change in the conductivity of the $\mathrm{VO}_{2}$ film, which demonstrates the conducting state at $T=348 \mathrm{~K}$. The transmission characteristic measured for this temperature is shown in Fig. 4c by black solid line. As is seen, the bandwidth of the transmission characteristic narrows and has the minimum loss level of $41.5 \mathrm{~dB}$ (see red long dash line in Fig. 4).

Description of the observed effect physical mechanism and numerical calculations of the transmission characteristics of the $\mathrm{YIG}-\mathrm{VO}_{2}$ regular structure were performed using the theory presented in our earlier works $[36,37]$. The theory describes the spin-wave propagation in heterostructures composed of an infinite number of ferrite, dielectric, and conductive layers. According to the theory, the complex dispersion relation for the YIG-VO $\mathrm{VO}_{2}$ bilayer $\omega(\boldsymbol{k})$ was found, where $\boldsymbol{k}=k^{\prime}+j k^{\prime \prime}$ is a complex propagation constant, $k^{\prime}$ and $k^{\prime \prime}$ are the phase and attenuation constants, respectively. The obtained phase constant was used for calculation of the phase shift of the microwave signal $\varphi=k^{\prime} l$ transmitted through the bilayered structure with a length $l$, while the attenuation constant was used for calculation of the power transmission coefficient $H p=20 \log \left(\exp \left(-k^{\prime \prime} l\right)\right)$. The characteristic feature of this model is that the power transmission coefficient for the $\mathrm{YIG}-\mathrm{VO}_{2}$ bilayered structure is obtained taking into account both the YIG-film saturation magnetization and the $\mathrm{VO}_{2}$-film conductivity. For the computer modeling, the values of the $\mathrm{VO}_{2}$ conductivity $\sigma(T)$ and the YIG-film saturation magnetization $M_{\mathrm{s}}(T)$ were calculated at a given temperature in accordance with Eq. (1) and Eq. (2), respectively. The dashed magenta lines in Fig. 4 represent the calculated transmission characteristics of the $\mathrm{YIG}-\mathrm{VO}_{2}$ structure for the temperature values used in the experiments. A deviation of the experimental dependences from the theoretical ones is explained by existence of additional sources of the insertion losses, such as the mismatch losses due to the reflection of the incident wave from the edges of the bilayered structure as well as the microstrip antenna impedance mismatches. 
(b) Microwave measurements of a magnonic crystal composed of the $\mathrm{YIG}^{\mathrm{V}} \mathrm{VO}_{2}$ bilayer

Consider here the magnonic crystal composed of the $\mathrm{VO}_{2}$ and the thickness-modulated YIG films as specified in Section II and in Fig. 1b. The transmission characteristics of the proposed MC measured for three temperatures are presented in Fig. 5a, Fig. 5b, and Fig. 5c.
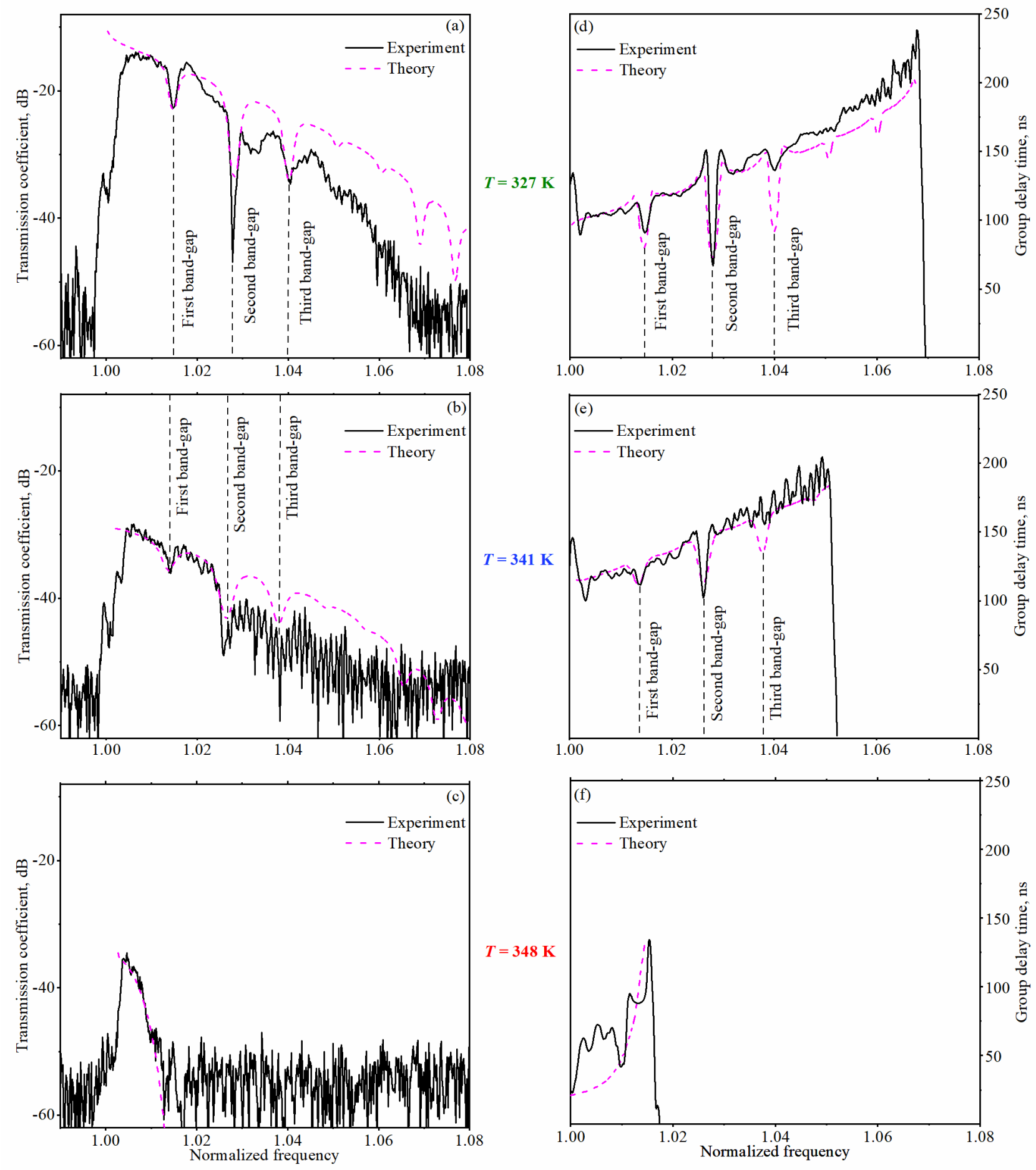
Fig. 5. Experimental (solid black lines) and theoretical (dashed magenta lines) transmission characteristics of the MC composed of $\mathrm{YIG}-\mathrm{VO}_{2}$ bilayer for three temperatures of (a) $T=327 \mathrm{~K}$, (b) $T=341 \mathrm{~K}$, and (c) $T=348 \mathrm{~K}$. Experimental (solid black lines) and theoretical (dashed magenta lines) dependences of the group delay time versus frequency for three temperatures of (d) $T=327 \mathrm{~K}$, (e) $T=341 \mathrm{~K}$, and (f) $T=348 \mathrm{~K}$.

In contrast to the regular waveguide, the transmission characteristic of the $\mathrm{MC}$ composed of the YIG- $\mathrm{VO}_{2}$ bilayer is more complicated. As is seen in Fig. 5a, at initial temperature $T=327 \mathrm{~K}$ the bandgaps are formed at the frequencies expected from the conventional Bragg analysis. In this case, the $\mathrm{VO}_{2}$ film has its original insulating state (see Fig. 2) and, therefore, has a negligible effect on the spin-wave propagation in the structure. As was discussed above, heating of the $\mathrm{YIG}^{-\mathrm{VO}_{2}}$ bilayer generally manifests itself in two effects. The first one is a reduction of the YIG-film saturation magnetization $M_{\mathrm{s}}(T)$; the second one is an increase of the $\mathrm{VO}_{2}$ conductivity. On the one hand, a change of $M_{\mathrm{s}}(T)$ provides a frequency shift of the transmission characteristic and has a negligible effect on a level of SW propagation losses in the experimental frequency range (see Fig. 3). On the other hand, a variation of the $\mathrm{VO}_{2}$ conductivity governs the spin-wave group velocity and defines a minimum $\mathrm{SW}$ attenuation in the layered structure (see Fig. 4). To investigate an influence of the second effect on the spin-wave dynamics, the SW carrier frequency was normalized by $f_{\perp}$ in Fig. 5. This enables one to keep out of sight the frequency shifts of the band-gap positions due to $M_{\mathrm{s}}(T)$ changing and analyze only the direct MIT impact on the original band structure of the $\mathrm{YIG}^{-\mathrm{VO}_{2}}$ bilayer MC. An increase in temperature up to $T=341 \mathrm{~K}$ providing the $\mathrm{VO}_{2}$ conductivity of $890 \Omega^{-1} \mathrm{~cm}^{-1}$ has still a weak impact on the frequency positions of the band-gaps (see Fig. 5b). At the same time, it leads to an enhancement of a SW attenuation around $14 \mathrm{~dB}$ in the band-gaps as well as in the pass-bands. 
In the same way as for a regular structure, an increase of temperature up to $T=348 \mathrm{~K}$ provides a sharp jump of the $\mathrm{VO}_{2}$ conductivity up to $\sigma=1380 \Omega^{-1} \mathrm{~cm}^{-1}$. It leads to a change in the spin-wave group velocity in the YIG film and narrows the transmission characteristic frequency bandwidth (see Fig. 5c). The bandwidth becomes less than the frequency distance to the first-order rejection band. As a result, the band structure of the proposed MC is fully suppressed.

In order to demonstrate that the observed effect is caused by the MIT phenomenon, the group delay time was measured as $\tau(f)=\frac{d \varphi}{d \omega}$. Here, $\varphi$ is the phase shift of the signal on the particular angular frequency $\omega$. The group delay time and complex $S_{21}$ characteristic were taken simultaneously using software embedded in the VNA R\&S®ZVA40. The obtained results are shown in Fig. 5d, Fig. 5e, and Fig. $5 \mathrm{f}$ by solid black lines, respectively. As is seen from Fig. 5d, at initial temperature of $T=327 \mathrm{~K}$ the group delay time starts from $\tau\left(f_{\perp}\right)=93 \mathrm{~ns}$ and increases up to $\tau\left(1.07 f_{\perp}\right)=225 \mathrm{~ns}$. This dependence demonstrates visible disturbances at the certain frequencies corresponding to the band-gaps of the MC. As is seen from Fig. 5e, a heating of the layered structure up to $T=341 \mathrm{~K}$ leads to a small increasing of $\tau\left(f_{\perp}\right)$ up to $114 \mathrm{~ns}$. This is due to a reduction in the SW group velocity, which is caused by decreasing of the YIG film saturation magnetization. Further heating of the bilayer up to $T=348 \mathrm{~K}$ leads to an increase in the $\mathrm{VO}_{2}$ conductivity that results in a drop of the group delay time, as shown in Fig. 5f. For example, the $\mathrm{MC}$ composed of the YIG film and the $\mathrm{VO}_{2}$ strip in a conducting state demonstrates a fourtime drop of $\tau(f)$ to $23 \mathrm{~ns}$ and a stronger frequency dependence, which provides $74 \mathrm{~ns}$ delay for $f=1.013 f_{\perp}$. It confirms a dominant role of the MIT effect over the temperature effect in formation of the MC frequency response.

Like in the previous Subsection, the experimental results were treated using the early-developed theory $[36,37]$. Theoretical characteristics were calculated by means of a two-step algorithm. At first step, the dispersion characteristics were found for SW propagating in the regular sections of the bilayer 
structure composed of the thin- or thick-YIG film. At the second step, the MC transmission characteristics and dependences of the group delay time were calculated by the transfer-matrix method $[26,43]$. Results of the theoretical analysis are shown in Fig. 5 by dashed magenta lines. The obtained results predict qualitatively the observed frequency dependences.

\section{DISCUSSION}

As shown in the previous Section, the group delay time of the surface SWs propagating in the YIG$\mathrm{VO}_{2}$ layered structures significantly changes in a relatively narrow temperature range from $327 \mathrm{~K}$ to $348 \mathrm{~K}$. A utilization of this phenomenon is practically beneficial because it enables one to control spinwave dynamics in various waveguiding structures. In particular, the low and high levels of microwave attenuation in the structures are determined by an insulating (i.e. high-resistance) and conducting (i.e. low-resistance) states of the $\mathrm{VO}_{2}$, respectively. As a result, the $\mathrm{YIG}-\mathrm{VO}_{2}$ layered structure operates as a switch. It means that the OFF and ON states are triggered by the MIT phase transition in the $\mathrm{VO}_{2}$ film, which is caused by temperature adjusting.

In order to demonstrate proof-of-concept switching capabilities for the proposed MC, a detailed numerical analysis of the dispersion characteristics of this structure was carried out. It is well known that the dispersion characteristics are uniquely related to the waveguiding transfer functions. While the real part of the wave-number defines the phase shift of the wave propagating in a waveguiding structure, its imaginary part describes the propagation loss. In this regard, an application of the dispersion characteristics to the description of the transmission coefficient and group delay characteristics benefits for a visualization of a transformation of the wave spectrum in the both $\mathrm{ON}$ and OFF states of the MC triggered by a rapid change in the $\mathrm{VO}_{2}$ conductivity. As in the experiments described in the previous Section, further numerical calculations were performed for the two temperatures of $327 \mathrm{~K}$ and $348 \mathrm{~K}$.

The calculated dispersion characteristics of the surface $\mathrm{SW}$ in the $\mathrm{YIG}-\mathrm{VO}_{2}$ based $\mathrm{MC}$ are presented in Fig. 6a and Fig. 6b by magenta solid lines. Here black dots show dispersion characteristics that were 
reconstructed from the experimentally taken phase-frequency characteristics. Magenta solid lines in Fig. 6c and Fig. 6d represent the theoretical dependences of group velocities, while black dots correspond to the group velocity obtained from the measurements.

Let us consider now the wave propagation in the $\mathrm{MC}$ at the initial temperature $T=327 \mathrm{~K}$ (see Fig. 5a and Fig. 5c). At this temperature, the $\mathrm{VO}_{2}$ film has a negligible impact on the spin-wave dynamics in the experimental frequency range. Therefore, the band structure of the thickness-modulated ferrite film is well defined. An interaction among the waves in the periodical sequence of the MC sections leads to a formation of the magnonic band-gaps at the wave-numbers $\boldsymbol{K}_{\mathrm{B} n}$ ( $n$ is a band-gap number) corresponding to the Bragg reflection law. It is clear that a band-gap with a finite frequency jump at the border of the Brillouin zone may be formed only in an idealistic loss-less artificial crystal. In this case, a total reflection of spin waves should be observed at the frequency ranges marked by shaded gray areas in Fig. 6a. Nevertheless, the dispersion characteristics remains continuous due to finite losses that are taken into account. Such a behavior is demonstrated in the inset of Fig. 6a. Fig. 6c shows the SW group velocity vs. frequency dependence in the proposed structure at initial temperature $T=327 \mathrm{~K}$. As is seen, the group velocity is sharply changed in the frequency ranges, where the slope of the spin-wave dispersion decreases, and has maxima at the frequencies corresponding to the Bragg resonance conditions (see the inset in Fig. 6c). 
(a)

(c)
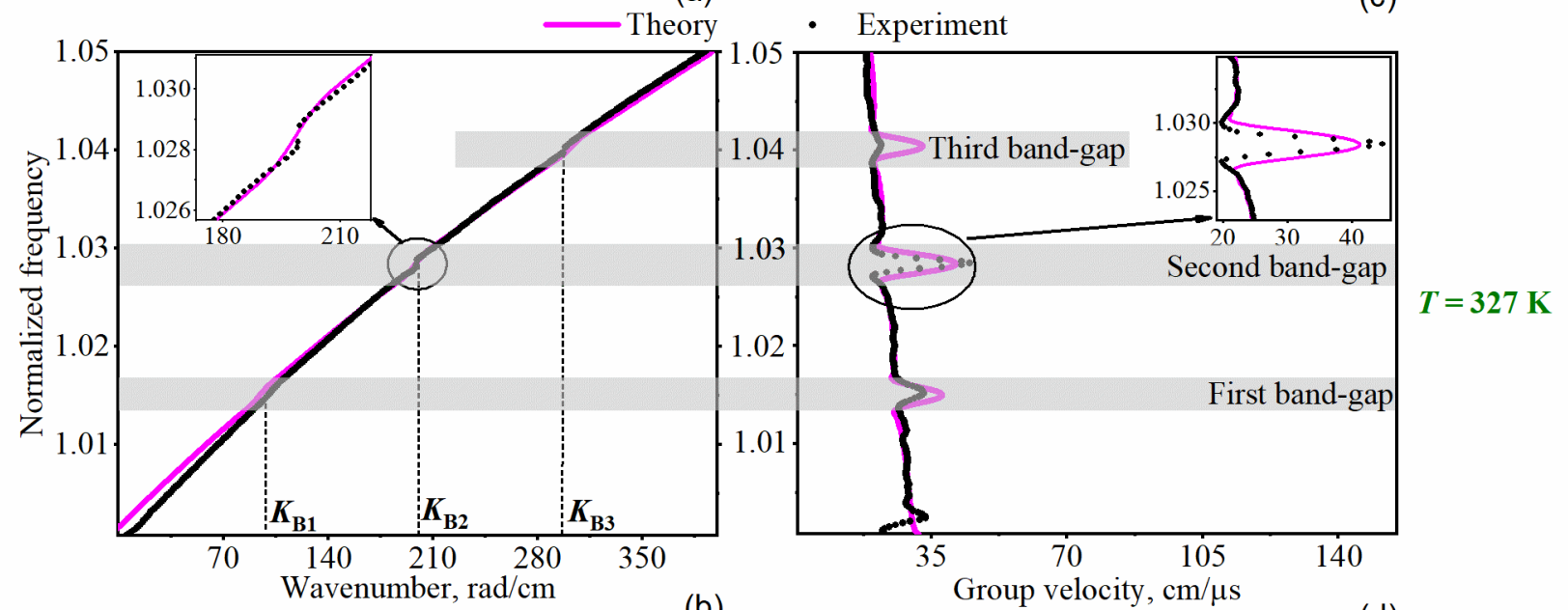

(b)

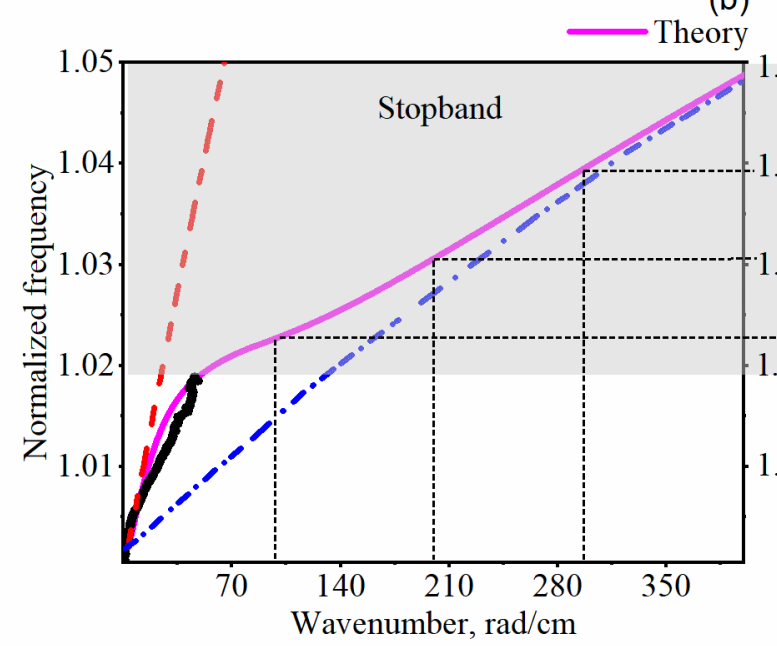

Group velocity, $\mathrm{cm} / \mu \mathrm{s}$

(d)

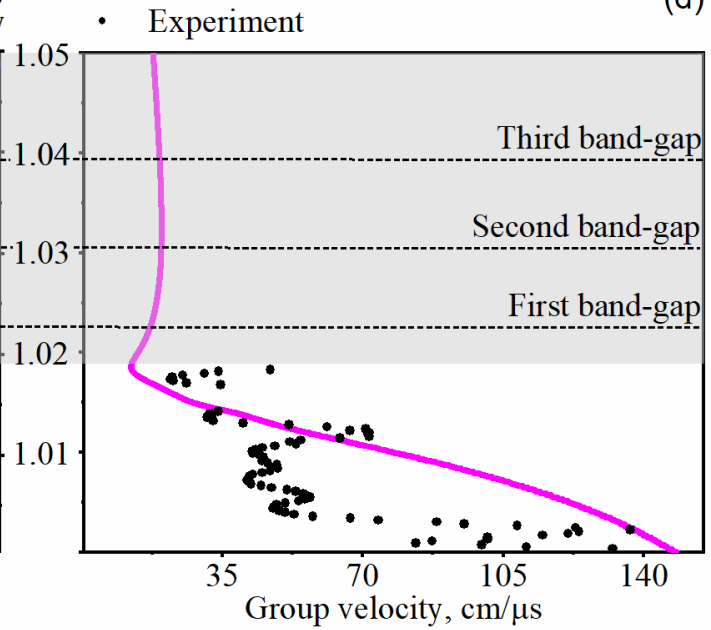

$T=348 \mathrm{~K}$

Fig. 6. Numerical analysis of the spin-wave dynamics in the $\mathrm{YIG}-\mathrm{VO}_{2}$ based MC. (a) The spectrum and (c) group velocity of the surface $\mathrm{SW}$ propagating in the $\mathrm{MC}$ at temperature $T=327 \mathrm{~K}$. Insets show peculiarities of the spin-wave dynamics in vicinity of the second band-gap. (b) The spectrum and (d) group velocity of the surface SW propagating in the heated MC at temperature $T=348 \mathrm{~K}$.

Let us turn now to changing in the band structure due to heating of the proposed MC up to $T=348$ $\mathrm{K}$. An increase in the $\mathrm{VO}_{2}$ conductivity considerably influences on the spin-wave dispersion, as shown in Fig. $6 \mathrm{~b}$ by magenta solid line. The dispersion characteristic can be divided into three frequency regions. For the frequencies within the first region $1<f / f_{\perp}<1.012$, the dispersion characteristic of the surface SW coincides with the dispersion characteristic of the spin wave in the YIG-film covered by a 
perfect metal shown by red dashed magenta line in Fig. 6b. After that, the calculated dispersion characteristic demonstrates transient behavior for the intermediate frequency region of $1.012<f / f_{\perp}<1.035$. Finally, for the frequency $f / f_{\perp}>1.035$ the dispersion characteristic of the wave in the proposed structure approaches to the dispersion characteristic of the spin wave in the uncovered YIG film shown by blue dash-dotted line in Fig. 6 b.

Fig. 6d shows the SW group velocity in the heated $\mathrm{YIG}-\mathrm{VO}_{2}$ based $\mathrm{MC}$. The value of the group velocity, obtained slightly above the $f_{\perp}$, is almost fourfold higher in comparison with the previous case. Nevertheless, in this case the value of the group velocity drops much more faster and its theoretical value reaches the minimum of $12 \mathrm{~cm} / \mu \mathrm{s}$ at $1.018 f_{\perp}$. Note that this point corresponds to the inflection point on the dispersion characteristic.

As is known, the SW propagation losses in the regular waveguiding structures is inversely relative to the group velocity [42]. The described behavior of the SW group velocity in the investigated structures determines growing of the propagation loss, which has a local maximum at $1.018 f_{\perp}$. Due to the Bragg condition the first band-gap should appear around $1.023 f_{\perp}$. However, this frequency is located in the frequency range of a full rejection of the spin waves, which corresponds to a stopband (see shaded gray area in Fig. 6b). It means that all band-gaps are suppressed.

\section{FINAL REMARKS AND OUTLOOKS}

The previous piece of the work has demonstrated as a proof-of-principle that thermally switching the $\mathrm{VO}_{2}$ on top of the YIG films by changing the temperature from $327 \mathrm{~K}$ to $348 \mathrm{~K}$, can considerably control the spin-wave group velocity. To accomplish this, the $\mathrm{YIG}-\mathrm{VO}_{2}$ bilayers have been placed in the temperature-controlled probe station. In spite of the fact that this method is suitable only for the laboratory experiments, the obtained findings suggest that the all-thin-film magnetic multilayers with vanadium dioxide are promising candidates for new functional devices. For practical applications, the 
method inducing the MIT phenomenon has to be optimized and improved in order to achieve faster change of the $\mathrm{VO}_{2}$ conductivity. Utilizing of the laser excitation with sufficient energy or electric fieldinduced MIT may be an elegant way, which would provide such optimization [38, 44].

Besides, we note that a proper choice of the dielectric thickness is one of the main questions arising out for the applications of the magnonic multilayers with vanadium dioxide. According to our estimates for the proposed $\mathrm{MC}$, a $200-\mu \mathrm{m}$-thick $\mathrm{SiO}_{2}$ layer is enough to minimize an influence of temperature on the YIG saturation magnetization without losing an efficiency of the reported suppression of the bandgap structure.

Let us consider now a switching time between $\mathrm{ON}$ and OFF states of the $\mathrm{YIG}-\mathrm{VO}_{2}$ bilayer. This time is defined by speed of a phase transition in a $\mathrm{VO}_{2}$ film from the insulator state to the conducting one. The experimental data show that the fundamental limit of the switching time in nanometer-thick $\mathrm{VO}_{2}$ films grown by magnetron sputtering is about $100 \mathrm{fs}$ [38]. The switching time for vanadium dioxide depends on various factors: a deposition technique employed in its fabrication, a thickness of the film, its crystalline state, a level of doping, and non-stoichiometry. These factors can be summarized as follows:

- A single-crystalline dioxide vanadium demonstrates more promising MIT properties than its polycrystalline counterpart due to narrower metal-insulator phase transition. Moreover, the epitaxial $\mathrm{VO}_{2}$ thin films grown on a dielectric substrate demonstrate a MIT at a lower temperature in comparison with bulk or polycrystalline materials [41];

- Small stoichiometric deviations destroy the sharpness of the metal-insulator phase transition and increase the hysteresis width. This provides an increase of the switching time between ON and OFF states, as well as the reset time back to ON state. Therefore, the oxygen deficiency, as well as the presence of impurities in a $\mathrm{VO}_{2}$ film limits an operation speed;

- The maximum operation speed, as well as the device structure miniaturization can be achieved by means of diminishing of $\mathrm{VO}_{2}$ film thickness. Herewith, it is necessary to take into account that below 
a certain characteristic $\mathrm{VO}_{2}$ film thickness the phase transition turns out to be impossible. Moreover, a significant decrease of thickness provides loss in efficiency of the described tuning mechanism. Thus, there is a trade-off between the switching time and the structure miniaturization.

\section{CONCLUSION}

We have proposed for the first time to employ the strongly correlated transition metal oxides to modify microwave properties of the ferrites to render novel functionalities of microwave devices. The experimental results describing particularities of the spin-wave dynamics in the structures composed of the vanadium dioxide and the yttrium-iron garnet films have been presented. It was shown that the metal-insulator phase transition in a $\mathrm{VO}_{2}$ film provides a new opportunity for control the spin-wave dynamics in a YIG film using a small temperature variation. According to the obtained results, a heating of the YIG-VO $\mathrm{VO}_{2}$ bilayer only by $20 \mathrm{~K}$ near the $\mathrm{VO}_{2}$ phase transition temperature leads to growing of the minimum loss level of spin waves by four orders of magnitude. From the point of view of applications, the described technique provides a novel tuning mechanism for the performance characteristics of magnetic multilayers.

An influence of the MIT phase transition in vanadium dioxide on the spin-wave dynamics in the periodic multilayers has also been investigated. In particular, it was found that an abrupt increase of the $\mathrm{VO}_{2}$ conductivity provided altering of the frequency response of the magnonic crystal from an original band structure to a full rejection of spin waves. In order to clarify the physical reasons of this behavior, a theoretical analysis of wave dynamics in the $\mathrm{YIG}-\mathrm{VO}_{2}$ bilayer has been carried out. It was found out that a heating of the magnonic crystal from $327 \mathrm{~K}$ up to $348 \mathrm{~K}$ lead to suppression of all band-gaps due to formation of the wide stopband where a full rejection of the spin waves is observed. This peculiarity is associated with a minimum of the SW group velocity and caused by increasing in losses with frequency due to an influence of the $\mathrm{VO}_{2}$ film. 
Apart from a controllable spin-wave intensity, the waveguiding structures composed of the YIG- $\mathrm{VO}_{2}$ bilayers may also offer the essential advantages, such as small sizes, well-developed techniques of fabrication, and ultrafast switching time. Therefore, it would be of a practical benefit to use heterogeneous ferrite-vanadium dioxide structures as an experimental testbed for investigations of the novel physical phenomena in the modern field of spin caloritronics addressing the interplay between spin and electron subsystems of solid materials.

\section{DATA AVAILABILITY}

The data that support the findings of this study are available within the article.

\section{REFERENCES}

1. V. V. Kruglyak, S. O. Demokritov, and D. Grundler, J. Phys. D: Appl. Phys. 43, 264001 (2010).

2. M. Krawczyk, D. Grundler, J. Phys. Condens. Matter 26, 123202 (2014).

3. A. V. Chumak, A. A. Serga, B. Hillebrands, J. Phys. D 50, 244001 (2017).

4. G. Csaba, Á. Papp, W. Porod, Phys. Lett. A 381, 1471-1476 (2017).

5. B. W. Zingsem, T. Feggeler, A. Terwey, S. Ghaisari, D. Spoddig, D. Faivre, R. Meckenstock, M. Farle, and M. Winklhofer, Nat. Commun. 10, 1-8 (2019).

6. A. B. Ustinov, A. V. Drozdovskii, B. A. Kalinikos, Appl. Phys. Lett. 96, 142513 (2010).

7. M. Inoue, A. Baryshev, H. Takagi, P. B. Lim, K. Hatafuku, J. Noda, and K. Togo, Appl. Phys. Lett. 98, 132511 (2011).

8. S. Atalay, A. O. Kaya, V. S. Kolat, H. Gencer, and T. Izgi, J. Supercond. Nov. Magn. 28, 20712075 (2015).

9. S. V. Grishin, E. N. Beginin, M A. Morozova, Y. P. Sharaevskii, and S. A. Nikitov, IEEE Trans. Magn. 50, 1-4 (2014).

10. E. Bankowski, T. Meitzler, R. S. Khymyn, V. S. Tiberkevich, A. N. Slavin, and H. X. Tang, Appl. Phys. Lett. 107, 122409 (2015).

11. A. A. Nikitin, A. B. Ustinov, A. A. Semenov, A. V. Chumak, A. A. Serga, V. I. Vasyuchka, E. Lähderanta, B. A. Kalinikos, and B. Hillebrands, Appl. Phys. Lett. 106, 102405 (2015).

12. A. V. Chumak, A. A. Serga, and B. Hillebrands, Nat. Commun. 5, 1-8 (2014).

13. P. Frey, A. A. Nikitin, D. A. Bozhko, S. A. Bunyaev, G. N. Kakazei, A. B. Ustinov, B. A. Kalinikos, F. Ciubotaru, A. V. Chumak, Q. Wang, V. S. Tiberkevich, B. Hillebrands \& A. A. Serga, Commun. Phys. 3, 17 (2020).

14. A. V. Chumak, T. Neumann, A. A. Serga, B. Hillebrands, and M. P. Kostylev, J. Phys. D 42, 205005 (2009). 
15. A. V. Chumak, V. S. Tiberkevich, A. D. Karenowska, A. A. Serga, J. F. Gregg, A. N. Slavin, and B. Hillebrands, Nat. Commun. 1, 141 (2010).

16. Q. Wang, A. V. Chumak, L. Jin, H. Zhang, B. Hillebrands, and Z. Zhong, Phys. Rev. B 95, 134433 (2017).

17. B. Rana, Y. Otani, Phys. Rev. Appl. 9, 014033 (2018).

18. A. B. Ustinov, A. V. Drozdovskii, A. A. Nikitin, A. A. Semenov, D. A. Bozhko, A. A. Serga, B. Hillebrands, E. Lähderanta, and B. A. Kalinikos, Commun. Phys. 2, 137 (2019).

19. M. Vogel, A. V. Chumak, E. H. Waller, T. Langner, V. I. Vasyuchka, B. Hillebrands, G. Von Freymann, Nat. Phys. 11, 487 (2015).

20. I. A. Golovchanskiy, N. N. Abramov, V. S. Stolyarov, P. S. Dzhumaev, O. V. Emelyanova, A. A. Golubov, V. V. Ryazanov, A. V. Ustinov, Adv. Sci. 6, 1900435 (2019).

21. A. B. Ustinov, B. A. Kalinikos, Tech. Phys. Lett. 40, 568-570 (2014).

22. M. A. Morozova, Y. P. Sharaevskii, and S. A. Nikitov, J. Commun. Technol. Electron. 59, 467-473 (2014).

23. I. A. Ustinova, A. A. Nikitin, and A.B. Ustinov. Tech. Phys. 61, 473-476 (2016).

24. A. A. Nikitin, A. A. Nikitin, A. V. Kondrashov, A. B. Ustinov, B. A. Kalinikos, and E. Lähderanta, J. Appl. Phys. 122, 153903 (2017).

25. A. A. Nikitin, A. A. Nikitin, A. B. Ustinov, E. Lähderanta, and B. A. Kalinikos, IEEE Trans. Magn. 54, 1-5 (2018).

26. A. A. Nikitin, A. A. Nikitin, I. L. Mylnikov, A. B. Ustinov, and B. A. Kalinikos, IET Microw. Antennas Propag. 14, 1304-1309 (2020).

27. G. Gubbiotti, X. Zhou, Z. Haghshenasfard, M. G. Cottam, and A. O. Adeyeye, Phys. Rev. B 97, 134428 (2018).

28. B. Rana, Y. Otani, Phys. Rev. Appl. 9, 014033 (2018).

29. E. N. Beginin, Y. A. Filimonov, E. S. Pavlov, S. L. Vysotskii, and S. A. Nikitov, Appl. Phys. Lett. 100, 252412 (2012).

30. N. Kanazawa, T. Goto, J. W. Hoong, A. Buyandalai, H. Takagi, and M. Inoue, J. Appl. Phys. 117, 17E510 (2015).

31. P. Kaboš, V.S. Stalmachov, Magnetostatic Waves and Their Application (Springer, New York, 1994).

32. M. Mruczkiewicz, M. Krawczyk, J. Appl. Phys. 115, 113909 (2014).

33. I. S. Maksymov, M. Kostylev, J. Appl. Phys. 116, 173905 (2014).

34. V. Flovik, B. H. Pettersen, and E. Wahlström, J. Appl. Phys. 119, 163903 (2016).

35. J. Trossman, J. Lim, W. Bang, J. B. Ketterson, and C. C. Tsai, J. Appl. Phys. 125, 053905 (2019).

36. A. A. Nikitin, V. V. Vitko, A. A. Nikitin, A. B. Ustinov, V. V. Karzin, A. E. Komlev, B. A. Kalinikos, and E. Lähderanta, IEEE Magn. Lett. 9, 3706905 (2018).

37. A. A. Nikitin, V. V. Vitko, A. A. Nikitin, A. B. Ustinov and B. A. Kalinikos, J. Phys. Conf. Ser. 1400, 044001 (2019).

38. Z. Yang, C. Ko, S. Ramanathan, Annu. Rev. Mater. Sci. 41, 337-367 (2011).

39. A. L. Pergament, G. B. Stefanovich, and A. Velichko, J. Select. Top. Nano Electron. Comput. 1, 2443 (2013).

40. S. D. Ha, Y. Zhou, A. E. Duwel, D. W. White, S. Ramanathan, IEEE Microw. Mag. 15, 32-44 (2014). 
41. R. Shi, N. Shen, J. Wang, W. Wang, A. Amini, N. Wang, and C. Cheng, Appl. Phys. Rev. 6, 011312 (2019).

42. D. D. Stancil and A. Prabhakar, Spin Waves: Theory and Applications (Springer, New York, 2009) 43. A. V. Chumak, A. A. Serga, B. Hillebrands, and M. P. Kostylev, Appl. Phys. Lett. 93, 022508 (2008).

44. B. G. Chae, H. T. Kim, D. H. Youn, and K. Y. Kang, Physica B Condens. Matter 369, 76-80 (2005). 


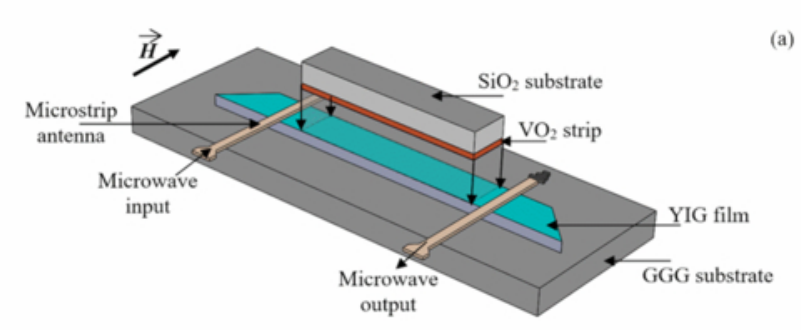

(a)
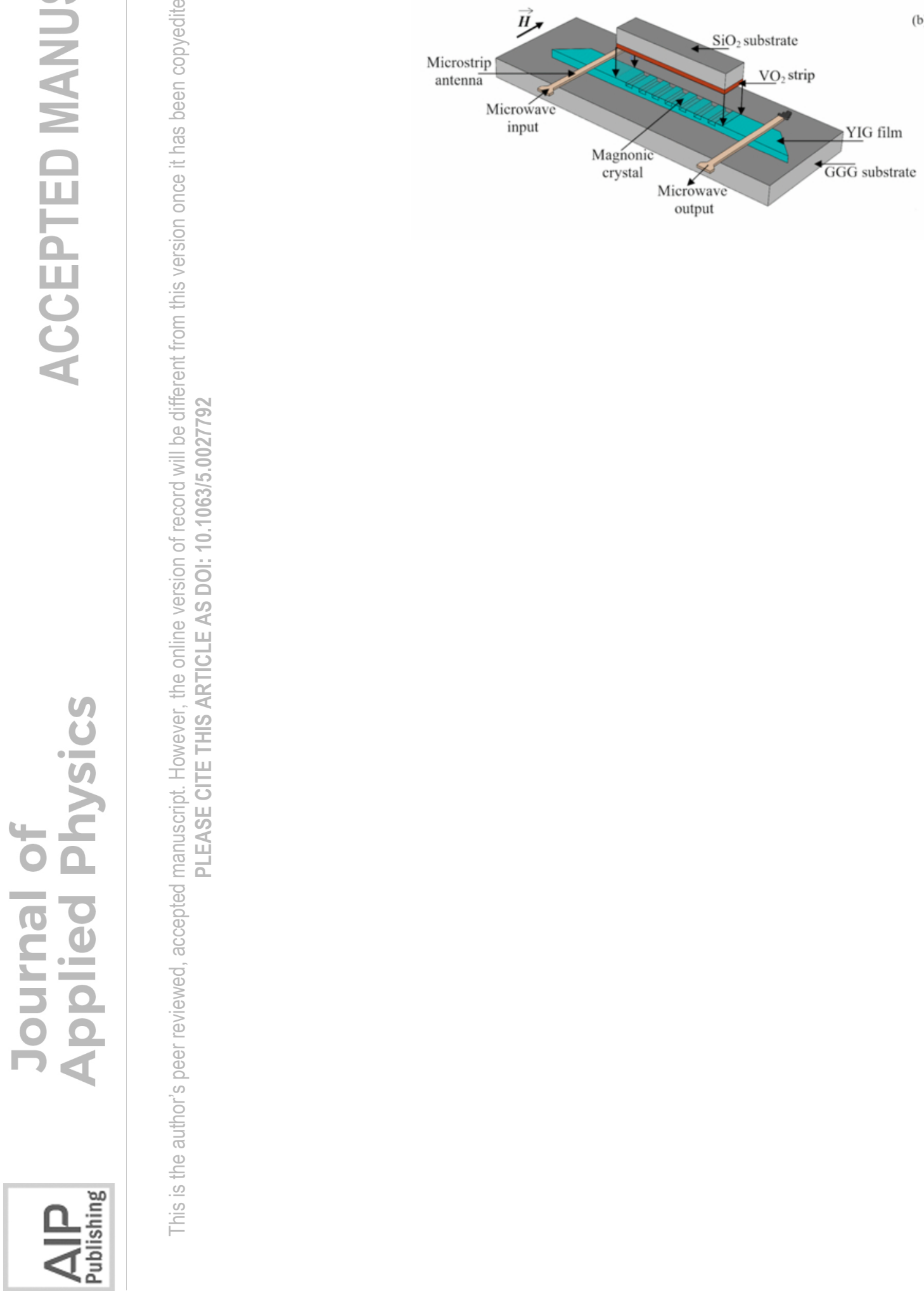
Insulating Intermediate state $_{1}$ Conducting

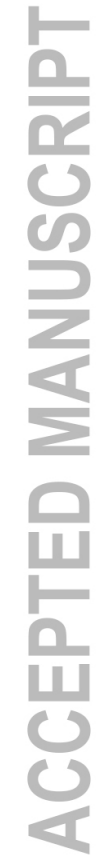

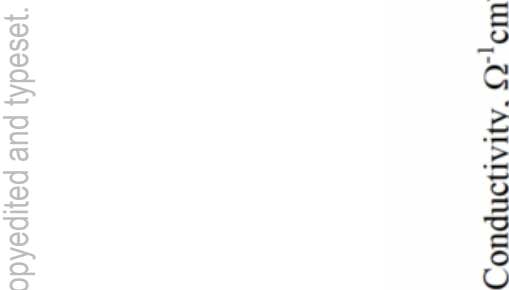
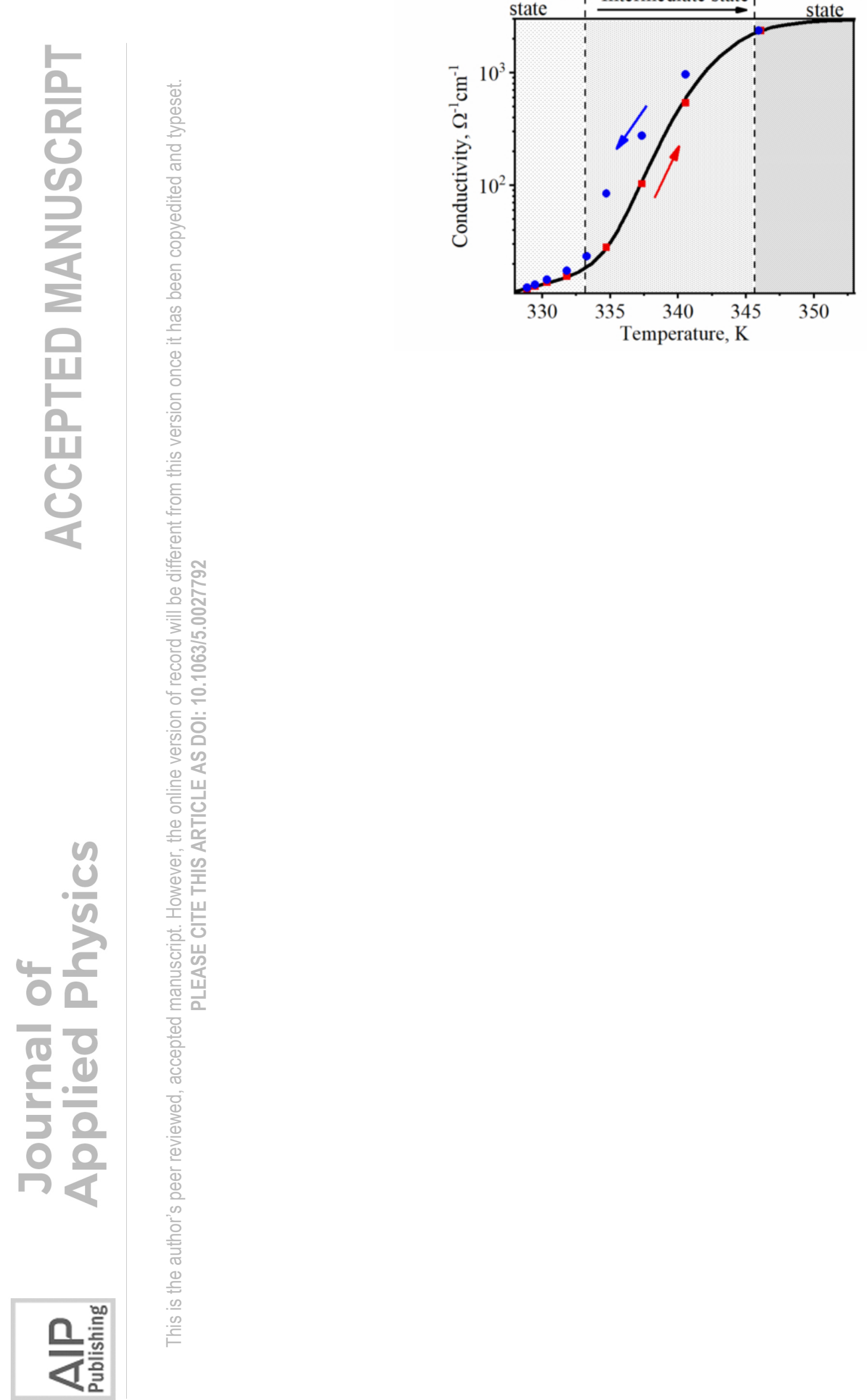


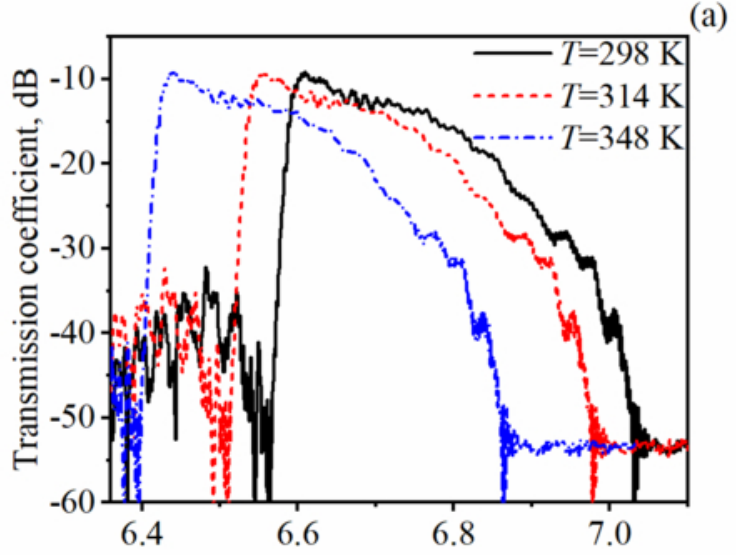

Frequency, GHz

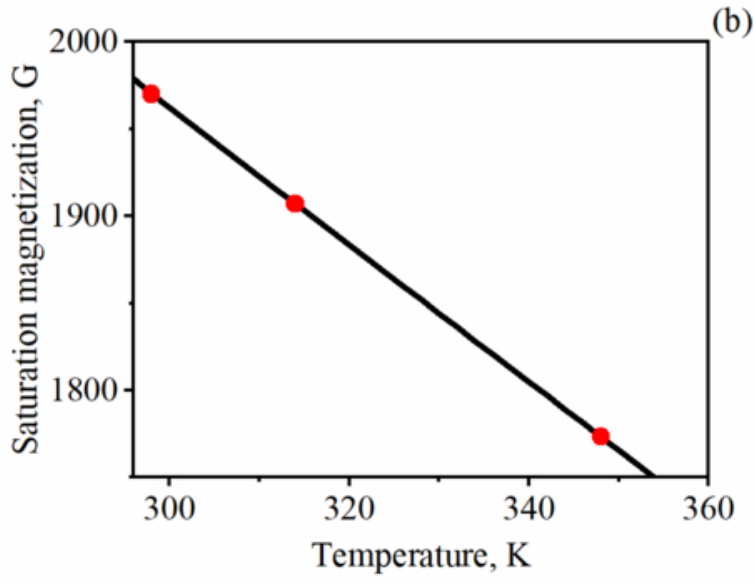


$T=327 \mathrm{~K}$

(a) (b)

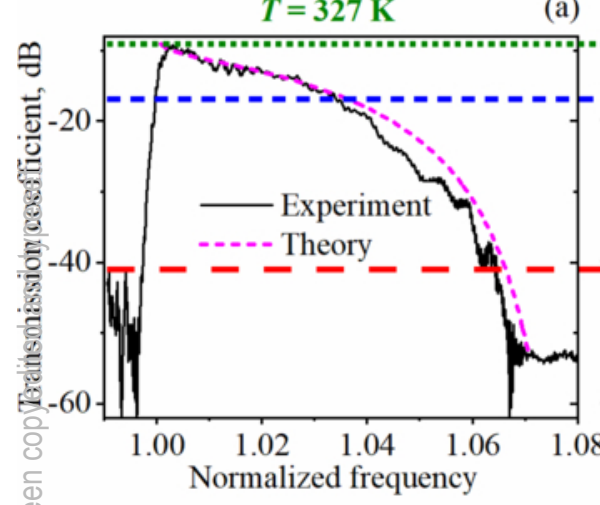

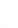

$T=348 \mathrm{~K}$

(c)

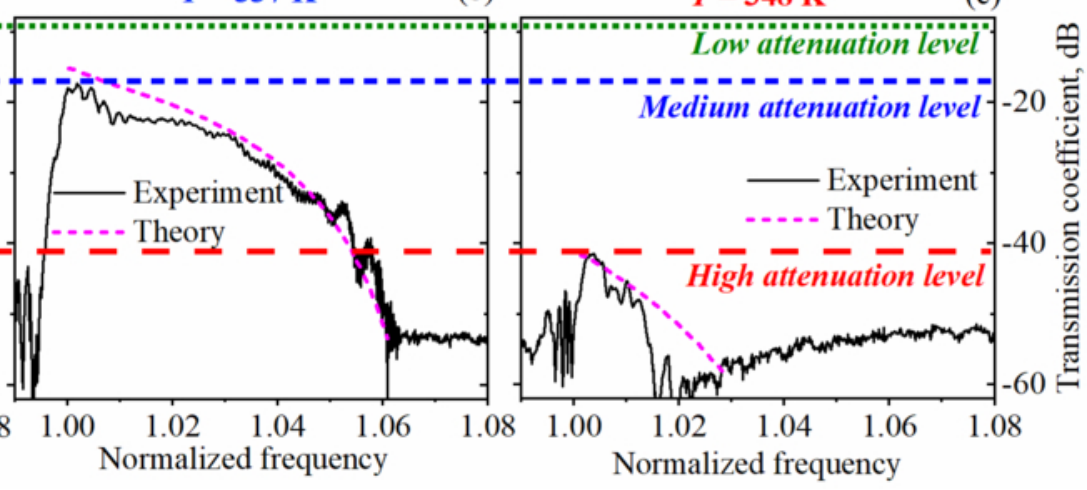




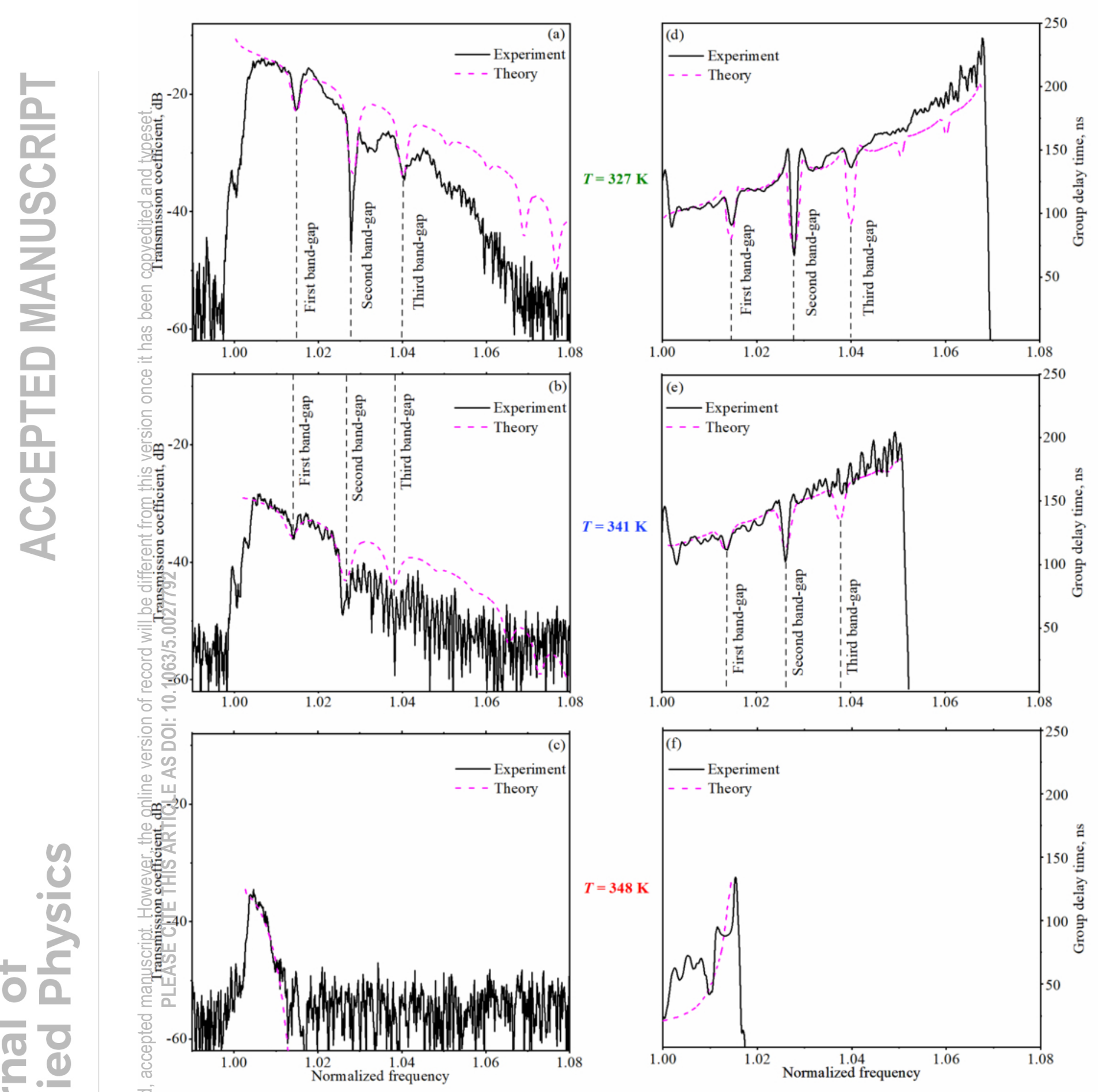




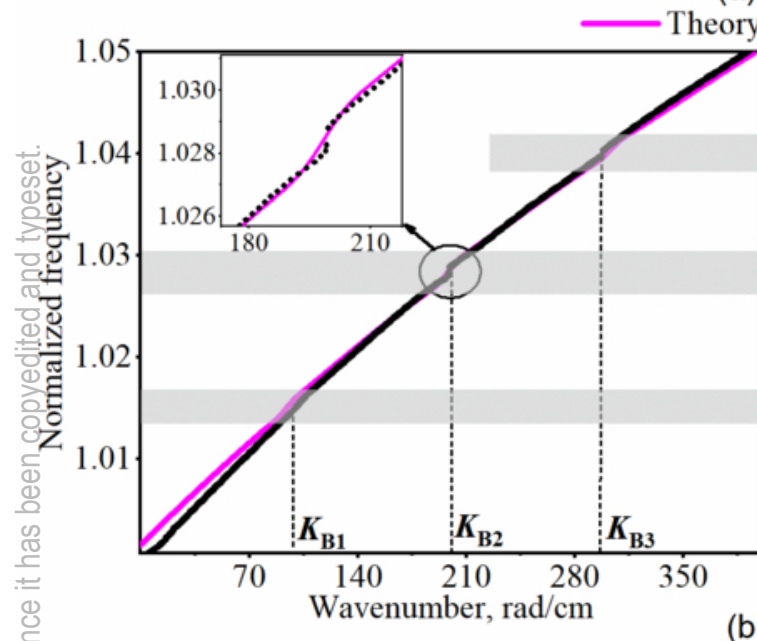

Experiment

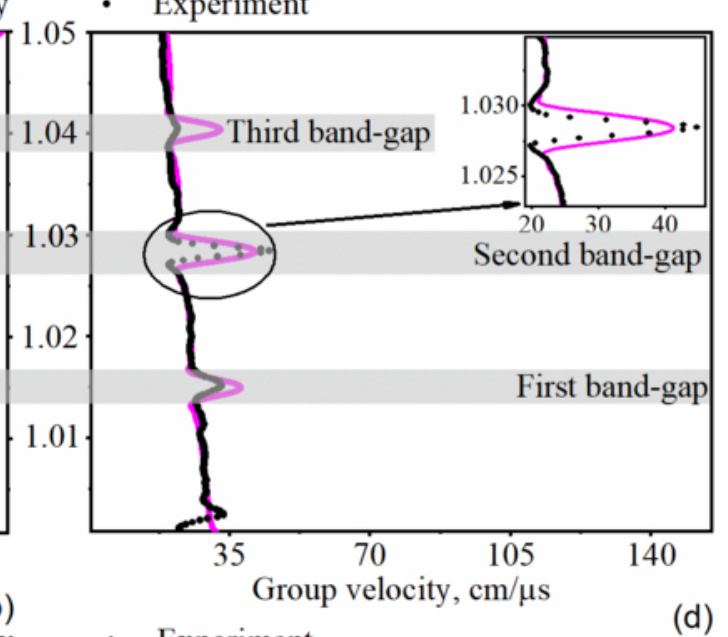

$$
T=327 \mathrm{~K}
$$

— Theory $\cdot$ Experiment

(d)
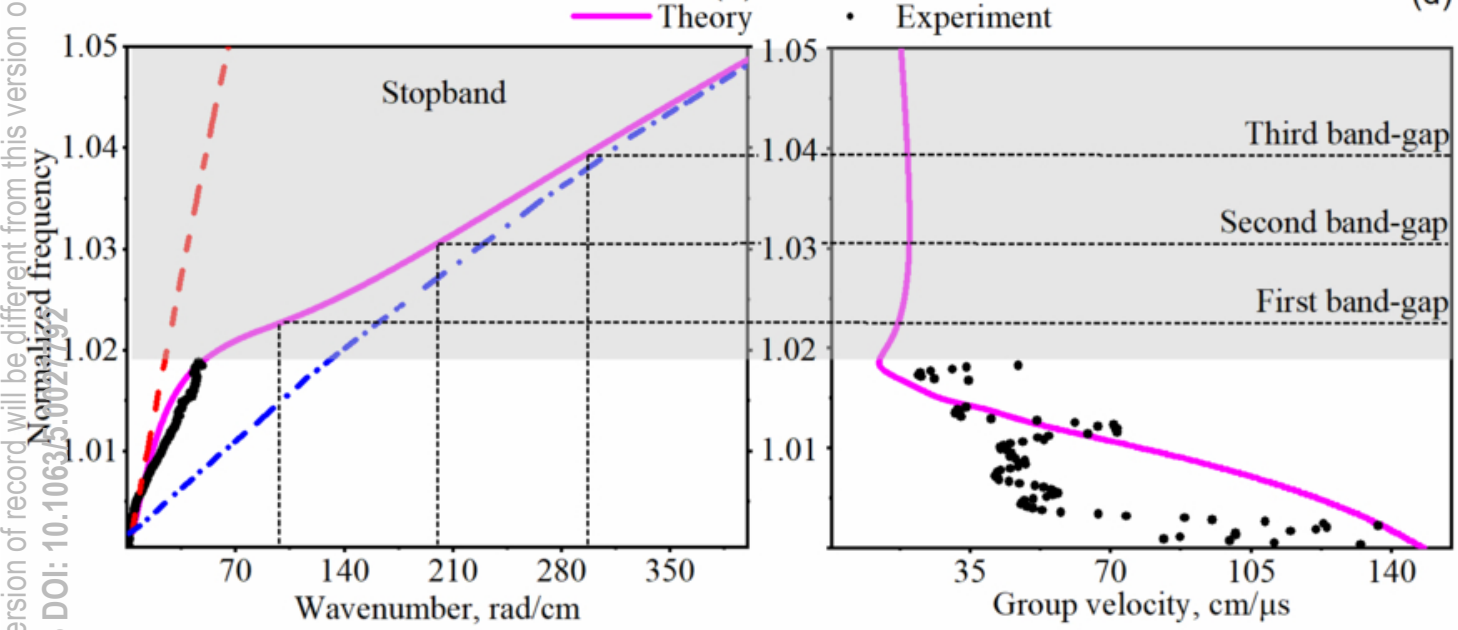

$T=348 \mathrm{~K}$ 